\title{
Scar Revision Surgery: The Patient's Perspective
}

\author{
Benjamin H Miranda, Anna Y Allan, Daniel P Butler, Paul D Cussons \\ Department of Plastic and Reconstructive Surgery, Royal Free London NHS Foundation Trust, Royal Free Hospital, London, UK
}

Background Insufficient satisfaction outcome literature exists to assist consultations for scar revision surgery; such outcomes should reflect the patient's perspective. The aim of this study was to prospectively investigate scar revision patient satisfaction outcomes, according to specified patient-selection criteria.

Methods Patients (250) were randomly selected for telephone contacting regarding scar revisions undertaken between 2007-2011. Visual analogue scores were obtained for scars preand post-revision surgery. Surgery selection criteria were; 'presence' of sufficient time for scar maturation prior to revision, technical issues during or wound complications from the initial procedure that contributed to poor scarring, and 'absence' of site-specific or patient factors that negatively influence outcomes. Patient demographics, scar pathogenesis (elective vs. trauma), underlying issue (functional/symptomatic vs. cosmetic) and revision surgery details were also collected with the added use of a real-time, hospital database.

Results Telephone contacting was achieved for 211 patients (214 scar revisions). Satisfaction outcomes were ' $2 \%$ worse, 16\% no change, and $82 \%$ better'; a distribution maintained between body sites and despite whether surgery was functional/symptomatic vs. cosmetic. Better outcomes were reported by patients who sustained traumatic scars vs. those who sustained scars by elective procedures $(91.80 \%$ vs. $77.78 \%, P=0.016)$ and by females vs. males $(85.52 \%$ vs. $75.36 \%, P<0.05)$, particularly in the elective group where males $(36.17 \%)$ were more likely to report no change or worse outcomes versus females $(16.04 \%)(P<0.01)$.

Conclusions Successful scar revision outcomes may be achieved using careful patient selection. This study provides useful information for referring general practitioners, and patient-surgeon consultations, when planning scar revision.

Keywords Patient satisfaction / Scarring / Standards / Plastic surgery / Patient selection
Correspondence:

Benjamin H Miranda

Department of Plastic and

Reconstructive Surgery, Royal Free

London NHS Foundation Trust, Royal

Free Hospital, London NW3 20G, UK

Tel: +44-7961-996-229

Fax: +44-20-7830-2468

E-mail: DrBMiranda@googlemail.

com

Received: 21 Apr 2015• Revised: 26 May $2015 \bullet$ Accepted: 6 Jul 2015

pISSN: 2234-6163 • elSSN: 2234-6171 • http://dx.doi.org/10.5999/aps.2015.42.6.729 • Arch Plast Surg 2015;42:729-734

No potential conflict of interest relevant to this article was reported.

\section{INTRODUCTION}

The ideal scar should be imperceptible, narrow, flat, homogenous, with no surrounding distortion or functional disturbance $[1,2]$. Factors affecting scar revision surgery may be classified as patient factors (age, comorbidity, medications, nutritional status, and smoking), local factors (blood supply, fluid collection, infection, and radiotherapy) or technical factors (technique, over mobile sites e.g., and joints) $[1,2]$. Tethered scars are a common surgical problem that may produce contour defects, lead to functional restriction or growth abnormalities [3]. To address such issues, many scar revision techniques have been described including excision and direct closure, dermis enhancement (dermal tube, double-breasting, and dermal graft), local flaps (Z-plasty, W-plasty, and other variants), fillers and tissue expansion [4-9]. However, regardless of the chosen surgical 
technique, unsatisfactory outcomes may still occur due to inadequate pre-revision critical analysis of the nature by which the scar was formed; our recommended critical analysis criteria are outlined (Table 1) $[2,10]$.

While a variety of scar assessment tools exist including the visual analogue scale (VAS), Vancouver burn scar assessment score and the clinical scar assessment scale, there is a shortfall of adequate literature data to assist the patient and surgeon in predicting scar revision outcomes $[4,11,12]$. Such data should primarily reflect the patient's perspective and recognise the natural history of scar maturation; it has been shown that when assessed at 6 and 18 months, patients recognise scar appearance improvements during maturation, a process that may take up to 2 years [13].

The primary aim of this study was to present patient satisfaction criteria, from 5 years of clinical practice at a large tertiary referral centre (Table 1). These departmental criteria reflect well recognised principles that directly apply to optimising patient selection for scar revision surgery (Table 1) [1,2,14-17]. They consider the 'presence' of sufficient time for scar maturation prior to revision, technical issues during or wound complications from the initial procedure that contributed to poor scarring, and 'absence' of site-specific or patient factors that negatively influence outcomes (Table 1) [1,2,14-17]. Further study aims included studying the differences between sex, pathogenesis (sustained in a traumatic vs. elective setting), underlying issue (func-

Table 1. Critical analysis criteria for scar revision patient selection

\begin{tabular}{|c|c|}
\hline $\begin{array}{l}\text { Critical analysis } \\
\text { criteria }\end{array}$ & Explanation \\
\hline Time & $\begin{array}{l}\text { Presence of sufficient time postoperatively to allow for } \\
\text { adequate scar maturation and final outcome observation } \\
\text { would favour a better outcome (up to } 2 \text { years). }\end{array}$ \\
\hline Technical issue & $\begin{array}{l}\text { Presence of an initial technical issue that could be } \\
\text { overcome during scar revision would favour a better } \\
\text { outcome e.g., tension, and poor alignment with skin } \\
\text { tension lines. }\end{array}$ \\
\hline Wound complication & $\begin{array}{l}\text { Presence of a postoperative wound complication that } \\
\text { contributed to poor scarring would favour a better } \\
\text { outcome e.g., dehiscence, infection, and fluid collection. }\end{array}$ \\
\hline Site-specific & $\begin{array}{l}\text { Absence of a site-specific reason that contributed to poor } \\
\text { scarring would favour a better outcome e.g., stretch due } \\
\text { to mobile areas/joints. }\end{array}$ \\
\hline Patient factors & $\begin{array}{l}\text { Absence of patient factors known to contribute to poor } \\
\text { scarring would favour a better outcome e.g., age, } \\
\text { comorbidity, medications, nutritional status, and smoking. }\end{array}$ \\
\hline \multicolumn{2}{|c|}{$\begin{array}{l}\text { These departmental criteria reflect well recognised principles that directly apply } \\
\text { to optimising patient selection for scar revision surgery; 'presence' of sufficient } \\
\text { time for scar maturation prior to revision, technical issues during or wound } \\
\text { complications from the initial procedure that contributed to poor scarring, and } \\
\text { 'absence' of site-specific or patient factors that negatively influence outcomes } \\
{[1,2,14-17] \text {. }}\end{array}$} \\
\hline
\end{tabular}

tional/symptomatic vs. cosmetic), and body sites. The null hypotheses were that no differences would be demonstrated.

\section{METHODS}

After clinical governance registration, patients were prospectively contacted by telephone, using a standardised protocol, to provide VAS satisfaction ratings (0-10; 0 , extremely bad scar; 5 , moderate scar; 10, excellent scar) of their scar, pre- and post- revision surgery. Patients (250/573) were randomly selected to ensure an adequate sample size for each of the following scar pathogenesis groups; traumatic vs. elective. The inclusion criterion was scar revision surgery undertaken between 2007-2011 to facilitate a minimum 2 years follow-up per patient. The exclusion criteria were scar revision operations for dog ears and patients who were not prospectively contactable. Further data were collected including patient demographics (age, sex), underlying issue (functional/symptomatic vs. cosmetic) and details of revision surgery (date, body site) were additionally cross referenced with a real-time secure plastic surgery electronic hospital database and electronic patient records. Parametric continuous data were analysed by t-tests and discrete data were analysed by chi-squared tests using SPSS ver. 20 (IBM Corp., Armonk, NY, USA).

\section{RESULTS}

Prospective telephone contacting was achieved for 211 patients (male $[\mathrm{M}]=68$ /female $[\mathrm{F}]=143$ ) who underwent 214 operations over the study period, with the proportion of operations performed by body site illustrated (Fig. 1). A minimum 2 years follow-up period was achieved for $100 \%$ of patient procedures $(214 / 214)$. Overall scar revision patient satisfaction outcomes were; $2.34 \%$ (5/214) worse, $15.89 \%$ (34/214) no change, $81.78 \%(175 / 214)$ better, distributions that remained similar regardless of body site (Fig. 2) or whether the underlying issue was functional/symptomatic vs. cosmetic (Table 2). In all cases where scar revision was performed for cosmetic reasons, the initial scarring was sustained in non-cosmetic procedures in patients without body dysmorphic disorder or related confounding diagnoses. When stratified by sex, females $(85.52 \%)$ were more likely than males $(75.36 \%)$ to report better satisfaction outcomes, hence males (24.45\%) were more likely to report worse or no change in outcome vs. females $(14.48 \%)(\mathrm{P}<0.05)$ (Table 2).

With respect to pathogenesis, 153 procedures were performed for $151(\mathrm{M}=47 / \mathrm{F}=104)$ patients (age $44 \pm 1$ years) whose scars were sustained in the elective setting, while 61 procedures were 


\section{Fig. 1. Proportion $(\%)$ and numbers of scar revision procedures $(n=214)$ undertaken by body site}

The proportion of procedures performed by body site were; head and neck (34.1\%, $n=73)$, chest and axilla $(23.8 \%, n=51)$, abdomen $(17.8 \%$, $n=38)$, pelvis and perineum $(1.4 \%, n=3)$, back $(2.3 \%, n=2)$, upper limb $(11.2 \%, n=24)$, lower limb and gluteal region $(9.3 \%, n=20)$.

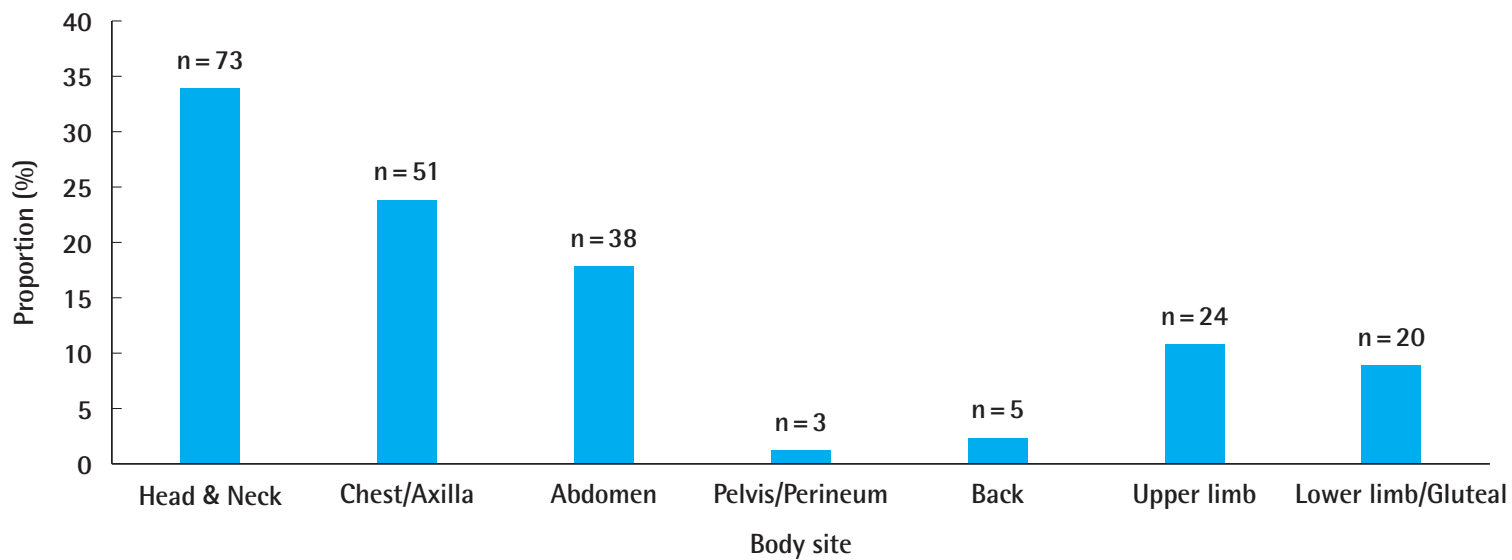

\section{Fig. 2. Scar revision patient satisfaction outcomes $(\%)$ by body site}

Overall scar revision patient satisfaction outcomes were as follows; 2.34\% (5/214) worse, 15.89\% (34/214) no change, 81.78\% (175/214) better, distributions that remained similar regardless of body site.

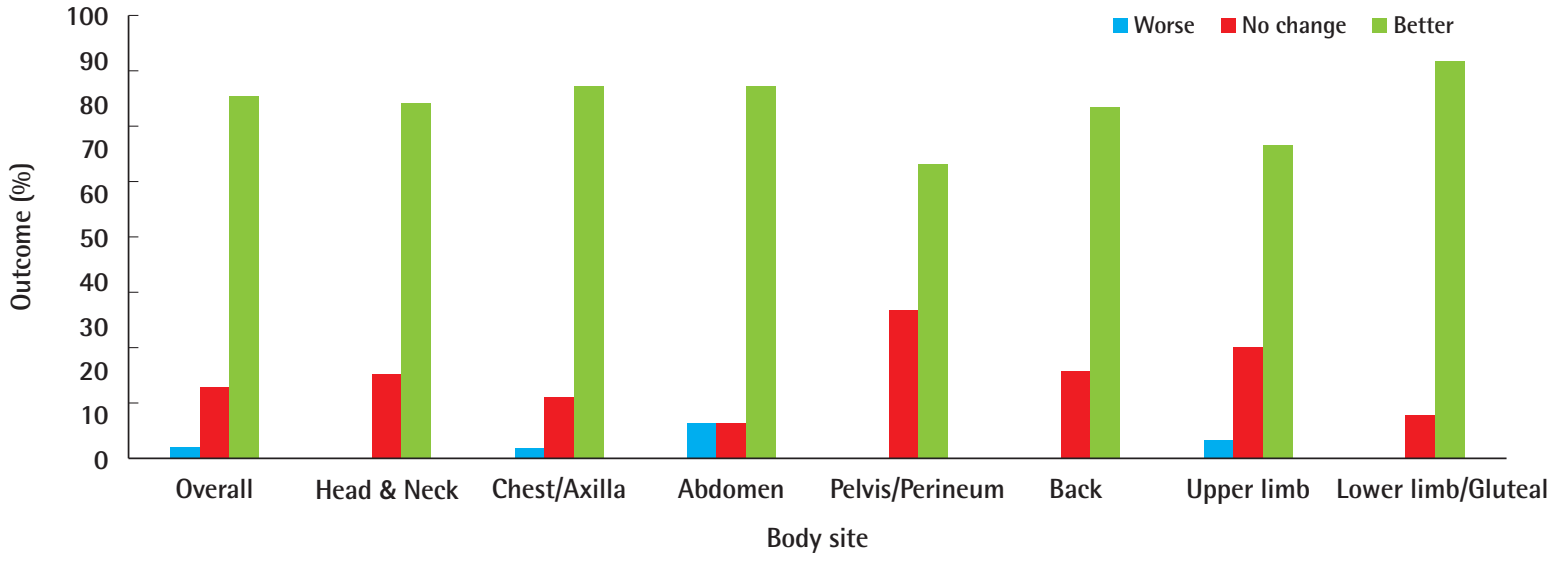

Table 2. Scar revision patient satisfaction outcomes stratified by sex, indication and pathogenesis

\begin{tabular}{|lccccccccc}
\hline Outcome (\%) & Male & Female & P-value & $\begin{array}{c}\text { Functional/ } \\
\text { symptomatic }\end{array}$ & Cosmetic & P-value & Elective & Trauma & P-value \\
\hline Worse & $4.35(3 / 69)$ & $1.38(2 / 145)$ & 0.18 & $3(3 / 100)$ & $1.75(2 / 114)$ & 0.55 & $2.61(4 / 153)$ & $1.64(1 / 61)$ & 0.67 \\
No change & $20.29(14 / 69)$ & $13.10(19 / 145)$ & 0.17 & $13(13 / 100)$ & $16.67(19 / 114)$ & 0.45 & $19.61(30 / 153)$ & $6.56(4 / 61)$ & $0.02^{*}$ \\
Better & $75.36(52 / 69)$ & $85.52(124 / 145)$ & $<0.05^{*}$ & $84(84 / 100)$ & $81.58(93 / 114)$ & 0.64 & $77.78(119 / 153)$ & $91.80(56 / 61)$ & $0.02^{*}$ \\
\hline *Significant $(P<0.05)$. & & & & & & &
\end{tabular}

performed for $60(M=21 / F=39)$ patients (age $40 \pm 2$ years) whose scars were sustained by trauma; both groups were agematched $(\mathrm{P}=0.14)$. Patients who sustained scars from trauma (91.80\%) were more likely to report better scar revision satisfaction outcomes than those who sustained scars in the elective setting $(77.78 \%)(P=0.016)$, hence patients who sustained scars in the elective setting (22.22\%) were more likely to report no change or worse outcomes than those who sustained scars from trauma $(8.20 \%)(\mathrm{P}=0.016)$. Furthermore, patients who sustained scars in the elective setting (19.61\%) were more likely 


\section{Fig. 3. Scar revision patient satisfaction outcomes for scars pathogenesis (elective vs. trauma), stratified by body site}

Patients who sustained scars from trauma (91.80\%) were more likely to report better scar revision satisfaction outcomes than those who sustained scars in the elective setting $(77.78 \%)(P=0.016)$, hence patients who sustained scars in the elective setting $(22.22 \%)$ were more likely to report no change or worse outcomes than those who sustained scars from trauma $(8.20 \%)(P=0.016)$. Patients who sustained scars in the elective setting $(19.61 \%)$ were more likely to report no change in outcome compared to those who sustained scars from trauma $(6.56 \%)(P=0.02)$. Worse scar revision outcomes were similar for those sustained in the elective setting vs. trauma $(2.61 \%$ vs. $1.64 \%$, $P=0.67)$. These distributions remained similar regardless of body site. $E$ (blue), patient group with scars sustained from elective surgery; $T$ (red), patient group with scars sustained from trauma; H\&N, head \& neck; Ax, axilla; Abdo, abdomen; LL/Glut, lower limb/gluteal; UL, upper limb; Pelv/Perin, pelvis/perineum.
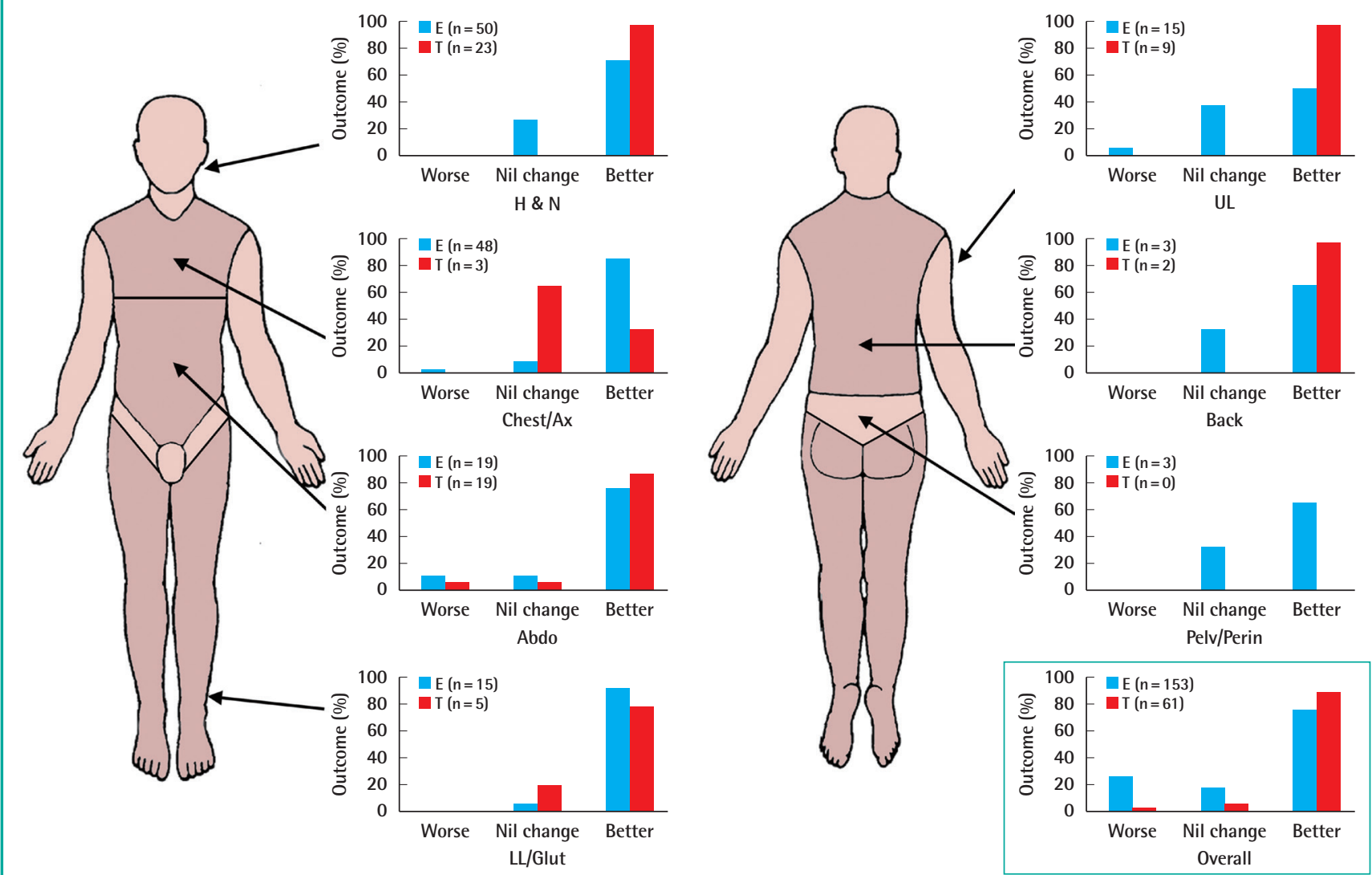

Table 3. Scar revision patient satisfaction outcomes for male and female patients who sustained scars in either an elective or traumatic setting

\begin{tabular}{|c|c|c|c|c|c|c|}
\hline \multirow{2}{*}{ Outcome (\%) } & \multicolumn{3}{|c|}{ Elective } & \multicolumn{3}{|c|}{ Trauma } \\
\hline & Male & Female & P-value & Male & Female & P-value \\
\hline Worse & $6.38(3 / 47)$ & $9.43(1 / 106)$ & 0.05 & $0(0 / 23)$ & $2.56(1 / 39)$ & 0.45 \\
\hline No change & $29.79(14 / 47)$ & $15.09(16 / 106)$ & $0.03^{*}$ & $0(0 / 23)$ & $7.69(3 / 39)$ & 0.18 \\
\hline Better & $63.83(30 / 47)$ & $83.96(89 / 106)$ & $<0.01^{\star *}$ & $100(23 / 23)$ & $89.74(35 / 39)$ & 0.11 \\
\hline
\end{tabular}

to report no change in outcome compared to those who sustained scars from trauma $(6.56 \%)(\mathrm{P}=0.02)$ (Table 2).

Pathogenesis sub-analysis by body site (Fig. 3 ) indicated similar scar revision outcome distributions as those previously illustrated (Fig. 2). However, due to relatively small sub-group sample sizes, only the head and neck region was further analysed, with patients who sustained scars from trauma (100\%) more likely to report better satisfaction outcomes than those who sustained scars in the elective setting $(72 \%)(\mathrm{P}<0.01)$. Pathogenesis sub-analysis by sex indicated that females (83.96\%) who sustained scars in the elective setting were more likely to report better outcomes than males $(63.83 \%)(\mathrm{P}<0.01)$, hence males (36.17\%) were more likely to report no change or worse outcomes versus females $(16.04 \%)(\mathrm{P}<0.01)$ (Table 2). Further- 
more, in the elective setting males $(29.79 \%)$ were more likely than females $(15.09 \%)$ to report no change in outcome $(\mathrm{P}=$ 0.03) (Table 2). No inter-sex differences were demonstrated in the traumatic setting (Table 2).

\section{DISCUSSION}

Patient-reported overall scar revision satisfaction outcomes generally followed a $2 \%$ worse, $16 \%$ no change, and $82 \%$ better distribution, figures that were maintained between body sites; the 3 most common body sites were head and neck (34\%), chest/ axilla (24\%) and abdomen (18\%) (Fig. 2). While scar revision has been associated with high patient satisfaction in the past, few studies have examined this objectively and from the patient's perspective [18]. Examples of such studies include an analysis of 18 ears in 13 patients, $84.62 \%$ reported good scar revision outcomes following intralesional excision of persistent hypertrophic or keloid scars, with the remaining 2 patients reporting acceptable outcomes (mean follow-up, 3.6 years); such findings are in-keeping with our results (Fig. 2) [19].

Another study of 32 patients, prospectively followed-up at 12 months after tethered scar revision using a dermal tube method, indicated $100 \%$ improvement outcomes from the patient's perspective using a VAS (0-2). Again, a high proportion of high satisfaction outcomes were reported; although higher than our findings, we present data from a broader spectrum of indications, with a larger sample size than previous studies, and over a minimum 2 years follow-up period to allow for scar maturation [4].

Not only were scar revision patient satisfaction outcomes similar regardless of whether surgical indications were for function$\mathrm{al} /$ symptomatic or cosmetic reasons, this distribution paralleled our previously discussed overall outcomes. The consistency between these findings provides supportive evidence for our critical analysis criteria for scar revision patient selection (Table 1); using these selection criteria should therefore facilitate more predictable outcome discussions, from the patient's perspective, during preoperative consultations.

The fact that patients who sustained scars from trauma were more likely to report better scar revision outcomes than those who sustained scars in the elective setting, and that those who sustained elective procedure scars were more likely to report no change or worse outcomes than those who sustained traumatic scars, is interesting (Table 2). Traumatic conditions are likely less favourable for initial good scar formation e.g., due to contamination, skin loss or because traumatic lacerations often do not respect the orientation of skin tension lines. Hence, it is probable that patients who sustain scars from trauma, are more likely to occupy more critical analysis patient selection criteria, favouring better scar revision outcomes (Table 1). Furthermore, such patients may also have lower expectations versus those who sustain scars in the elective setting, thus influencing better reported outcomes following scar revision surgery.

Of further interest, females $(85.52 \%)$ were more likely to report better outcomes versus males $(75.36 \%)(\mathrm{P}<0.05)$, a trend that was sustained for scars sustained in the elective setting ( $83.96 \%$ vs. $63.83 \%, \mathrm{P}<0.01$ ) but not during trauma; hence in the elective setting only, male patients were more likely to report worse or no change in outcome versus females (Tables 2, 3). Furthermore in the elective setting only, male patients were more likely to report no change in outcome vs. females (Table 3). Psychological observations of patients seeking elective cosmetic procedures have been reported in the literature since the 1940s [20]. At this time surgeons were warned of the psychopathology of male patients and the 'insatiable' surgery patient seeking multiple elective cosmetic procedures in pursuit of the perfect face; despite concerns regarding the psychological state of patients, procedures were rarely excluded and positive psychiatric outcomes reported [21]. Pre-selection criteria have evolved dramatically since this time, with emphasis on recognised psychological conditions e.g. body dysmorphic disorder and an increase in male uptake, in order to improve patient selection for elective cosmetic procedures [22-24]. These studies relate to our findings as they support the idea of inter-sex differences that may vary across a broad range of elective cosmetic procedures; our data support the concept of possible personality differences between females and potentially hyper-aware males, regarding the cosmetic appearance of their scars. Higher expectation precipitated by such personality traits could negatively influence the patient's opinion of their scar revision outcome. This is particularly so in the elective setting as patient expectation may be significantly higher.

In conclusion, this study demonstrates that successful scar revision surgery outcomes may be achieved using careful patient selection. The outlined patient selection criteria in this study directly apply to optimising patient selection for scar revision surgery; 'presence' of sufficient time for scar maturation prior to revision, technical issues during or wound complications from the initial procedure that contributed to poor scarring, and 'absence' of site-specific or patient factors that negatively influence outcomes (Table 1) [1,2,14-17]. Overall patient satisfaction outcomes were reported as; $2 \%$ worse, $16 \%$ no change and $82 \%$ better (Fig. 2). These figures were consistent between body sites and better outcomes were reported for revisions undertaken in scars sustained from trauma as opposed to elective procedures (Table 3, Figs. 2, 3). Overall, females reported better scar revision outcomes vs. males, particularly with scars sustained in the 
elective scenario where males were more likely to report worse/ no change in outcome (Tables 2,3). These evidence-based findings provide useful information for the referring general practitioner and patient-surgeon consultation when planning scar revision.

\section{REFERENCES}

1. Parkhouse N, Cubison TC, Humzah MD. Scar revision. In: Mathes SJ, Hentz VR, editors. Plastic surgery. 2nd ed. Philadelphia: Saunders; 2006. p.235-68.

2. Perez-Bustillo A, Gonzalez-Sixto B, Rodriguez-Prieto MA. Surgical principles for achieving a functional and cosmetically acceptable scar. Actas Dermosifiliogr 2013;104:17-28.

3. van Zuijlen PP, Angeles AP, Kreis RW, et al. Scar assessment tools: implications for current research. Plast Reconstr Surg 2002;109:1108-22.

4. Mallucci P, Abood A, Bistoni G. The dermal tube: a versatile tool in scar revision. J Plast Reconstr Aesthet Surg 2009;62: 1223-6.

5. Cavadas PC. The three-dimensional Z-plasty for the treatment of depressed adhered scars. Plast Reconstr Surg 2001; 107:1076-7.

6. Klein AW. Skin filling. Collagen and other injectables of the skin. Dermatol Clin 2001;19:491-508.

7. Fagien S, Elson ML. Facial soft-tissue augmentation with allogeneic human tissue collagen matrix (Demalogen and Dermaplant). Clin Plast Surg 2001;28:63-81.

8. McGregor AD, McGregor IA. 10th ed. London: Churchill Livingston; 2000.

9. Billings E Jr, May JW Jr. Historical review and present status of free fat graft autotransplantation in plastic and reconstructive surgery. Plast Reconstr Surg 1989;83:368-81.

10. Batra RS. Surgical techniques for scar revision. Skin Therapy Lett 2005; 10:4-7.

11. Sullivan T, Smith J, Kermode J, et al. Rating the burn scar. J Burn Care Rehabil 1990;11:256-60.

12. Beausang E, Floyd H, Dunn KW, et al. A new quantitative scale for clinical scar assessment. Plast Reconstr Surg 1998; 102:1954-61.
13. Martin D, Umraw N, Gomez M, et al. Changes in subjective vs objective burn scar assessment over time: does the patient agree with what we think. J Burn Care Rehabil 2003; 24:239-44.

14. Schweinfurth JM, Fedok F. Avoiding pitfalls and unfavourable outcomes in scar revision. Facial Plast Surg 2001;17: 273-8.

15. Broughton Gn, Crosby MA, Coleman J, et al. Use of herbal supplements and vitamins in plastic surgery: a practical review. Plast Reconstr Surg 2007;119:48e-66e.

16. Watson D, Reuther MS. Scar revision techniques-pearls and pitfalls. Facial Plast Surg 2012;28:487-91.

17. Krueger JK, Rohrich RJ. Clearing the smoke: the scientific rationale for tobacco abstention with plastic surgery. Plast Reconstr Surg 2001;108:1063-73.

18. di Summa PG, Wettstein R, Erba P, et al. Scar asymmetry after abdominoplasty: the unexpected role of seroma. Ann Plast Surg 2013;71:461-3.

19. Yang JY, Yang SY. Are auricular keloids and persistent hypertrophic scars resectable? The role of intrascar excision. Ann Plast Surg 2012;69:637-42.

20. Linn L, Goldman IB. Psychiatric observations concerning rhinoplasty. Psychosom Med 1949;11:307-14.

21. Gifford S. Cosmetic surgery and personality change: a review and some clinical observations. In: Goldwyn RM, Mimis C, editors. The unfavorable result in plastic surgery: avoidance and treatment. Boston: Little Brown; 1972. p.1133.

22. Picavet VA, Gabriels L, Grietens J, et al. Preoperative symptoms of body dysmorphic disorder determine postoperative satisfaction and quality of life in aesthetic rhinoplasty. Plast Reconstr Surg 2013;131:861-8.

23. Constantian MB. The new criteria for body dysmorphic disorder: who makes the diagnosis? Plast Reconstr Surg 2013;132:1759-62.

24. Barone M, Cogliandro A, Persichetti P. Preoperative symptoms of body dysmorphic disorder determine postoperative satisfaction and quality of life in aesthetic rhinoplasty. Plast Reconstr Surg 2013;132:1078e-1079e. 